\title{
Survival rate of colon and rectum cancer in Iran: A systematic review and meta-analysis
}

\author{
M. H. PANAHI ${ }^{1}$, H. PANAHI ${ }^{2}$, A. MAHDAVI HEZAVEH ${ }^{3}$, M. A. MANSOURNI ${ }^{1}$, R. BIDHENDI YARANDI ${ }^{1,4, *}$ \\ ${ }^{1}$ Department of Epidemiology and Biostatistics, School of Public Health, Tehran University of Medical Sciences, Tehran, Iran; ${ }^{2}$ Department of \\ Psychology, Lorestan University, Lorestan, Iran; ${ }^{3}$ Center for Non-Communicable Diseases, Deputy of Health, Ministry of Health and Medical \\ Education, Tehran, Iran; ${ }^{4}$ Reproductive Endocrinology Research Center, Research Institute for Endocrine Sciences, Shahid Beheshti University \\ of Medical Sciences, Tehran, Iran
}

${ }^{*}$ Correspondence: razi_bidhendi@yahoo.com

Received January 31, 2019 / Accepted April 10, 2019

\begin{abstract}
Colorectal cancer is one of the deadliest cancers worldwide. Effective screening, surveillance and prevention can decrease its incidence, mortality and burden. This meta-analysis aims to provide a pooled estimation of 5-year survival rate for colorectal cancer based on topography codes and treatment in Iranian population. A systematic search for literature was done in international and national databases up to July 2018. Twenty-seven studies from 4929 articles met the eligible criteria. The overall pooled 5-year survival rates of colorectal cancer, colon, rectal and sigmoid were 56\% (95\% CI: 49, 63), 53\% (95\% CI: 41, 65), 52\% (95\% CI: 41, 62) and 38\% (95\% CI: 22, 55), respectively. In addition, 5-year survival rate of colorectal cancer after surgery was $64 \%$ (95\%CI: 50,78$)$. Subgroup analysis by type of data source showed significantly higher rate of survival in oncology center $(29 \%)$ than hospital-based $(\mathrm{p}=0.005)$. As a conclusion, low survival rate of colorectal cancer in Iran necessitates effective screening and surveillance strategies to find precancerous polyps and detect early-stage cases with lower stage risk of cancer.
\end{abstract}

Key words: survival rate, colorectal neoplasms, meta-analysis

Cancer is one of the most important health problems in the world. In 2015, it was recognized as the second leading cause of death (8.8 million deaths) globally. Approximately, $70 \%$ of deaths from cancer occur in low-income and middleincome countries $[1,2]$. Colorectal cancer (CC) with 1.7 million new cases was reported as the second most prevalent cancer among men and the third among women. Its incidence increased by $37 \%$ throughout the world from 2005 to 2015 [2]. According to Iran National Cancer Registry in 2014, CC with the total incidence rate of 9861 cases in 30 Provinces of Iran (Qom Province excluded) and the age-standardized rate of 16.57 and 11.86 per 100,000 was the fifth most prevalent cancer among men and the second one among women. Different studies indicated an increase in the incidence of this disease in recent years in Iran [3-5]. Cancer survival rate (SR) is a widely used indicator for physicians, patients and health policymakers, and reflects the prognosis of cancer patients [6]. It is defined as a percentage of patients with specific cancer who survive after a certain period. In recent years, several studies have investigated the SR of CC patients in Iran. Despite numerous studies, no pooled estimation has yet been performed in this regard. The aims of this system- atic review and meta-analysis were to estimate the 5-year SR (5-YSR) of CC. The subgroup analysis based on the topography codes of $\mathrm{CC}$, the type of treatment and type of data source to identify the heterogeneity of the results have been performed as well.

\section{Materials and methods}

In this study, we applied the Preferred Reporting Items for Systematic reviews and Meta-Analyses (PRISMA) guidelines [7]. According to the classification provided by the International Agency for Research on Cancer (IARC), CC includes colon (C-18), sigmoid (C-19) and rectal cancers (C-20).

Information sources and search strategy. This study investigated the articles published using Iranian databases such as Irandoc, Magiran, SID, and international databases such as Scopus, Google Scholar, PubMed and Science Direct until July 2018. References were also investigated manually (with no limitation in terms of year of publication and language) to ensure that all relevant papers are obtained. We used the following strategy search in PubMed $((()(($ "Intestinal Neoplasms”[Mesh]) OR “Colorectal Neoplasms”[Mesh]) 


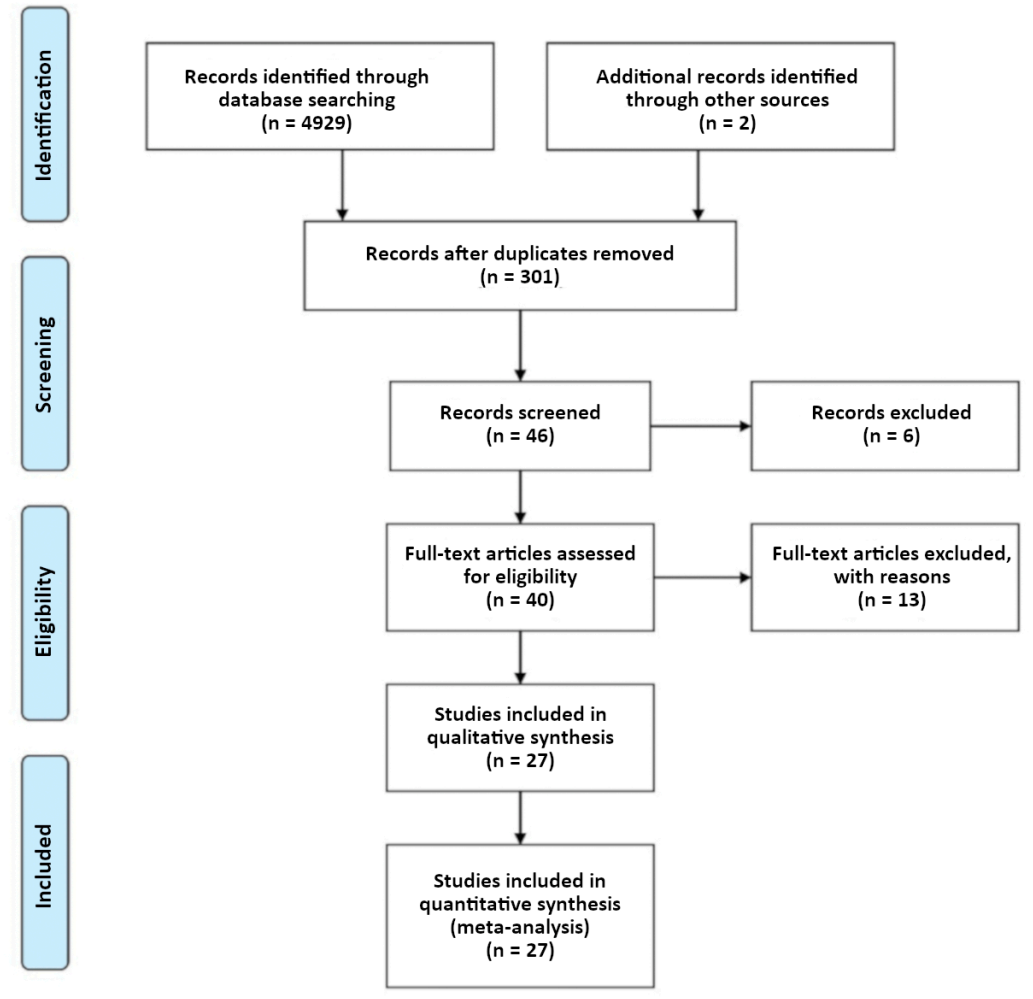

Figure 1. PRISMA flaw diagram

OR “Gastrointestinal Neoplasms"[Mesh]) OR "Rectal Neoplasms"[Mesh]) OR "Colonic Neoplasms"[Mesh])) AND Survival) AND Iran and equivalent strategy search in other databases.

Eligibility criteria for including the studies. All studies conducted on the SR of CC patients in Iran (cohort and crosssectional studies) regardless of the topography of cancer with one, two, three, four, five and ten-year SRs without any limitation were included in this study. Meta-analysis was done on studies with 5-YSR. Titles and abstracts were reviewed and irrelevant, duplicated papers and those with incomplete data were excluded.

Data extraction. Two investigators (MHP and HP) separately reviewed the titles and abstracts of all articles. In the next step, the full texts of the selected papers were studied using eligibility criteria and necessary data was extracted. Disagreements were solved by an independent third expert (MAM). Data were extracted from studies based on their lead author, date of the study, type of treatment, stage of disease, sample size, patients' age, and 1, 2, 3, 5 and 10-year SRs.

Statistical analysis. Meta-analysis was run to obtain pooled 5-YSR of specified outcomes. I-squared statistics were estimated as the measures of heterogeneity. Random-effect method was applied for values upper $50 \%$ otherwise fixedeffect method. Forest plot was also drawn to summarize the result of each study's effect sizes and its $95 \%$ confidence intervals (CIs).
Begg's test was used to assess publication bias, it was found significant for $\mathrm{p}$-value $<0.05$. Trim and fill method was conducted to correct for publication bias [8]. Furthermore, random effect meta-regression model was fitted to the estimate difference between studies with 5-YSR of "Postsurgery" and "Unrecognized treatment", Source of data including "Oncology center", "Cancer registry center" and "Hospital-based", and also to identify the sources of heterogeneity. Bubble plots were drawn to present the fitted models. Sensitivity analysis was conducted to explore the sources of heterogeneity and evaluating the results as well. Statistical analysis was done using STATA software (version 10; STATA, INC., College Station, TX, USA).

\section{Results}

Following the initial searches; 4,929 papers were extracted. Duplicated studies were excluded, titles and abstracts were reviewed, and finally, after the study of the full texts of papers, 27 papers (20 papers in English and 7 papers in Persian) were eligible for analysis [S1-S26, 9]. The study flow diagram was drawn following the PRISMA guideline statements (Figure 1).

Meta-analysis was conducted on these 27 papers. All studies that had investigated the SRs of patients with CC were included, regardless of the topography of cancer. Relevant data are presented in Table 1. 
Table 1. Characteristics of the included studies in systematic review of the SR of CC

\begin{tabular}{|c|c|c|c|c|c|c|c|c|c|c|c|c|}
\hline \multirow{2}{*}{$\begin{array}{l}\text { Ref. } \\
\text { No. }\end{array}$} & \multirow{2}{*}{$\begin{array}{l}\text { First author } \\
\text { (Publication year) }\end{array}$} & \multirow{2}{*}{ Type } & \multirow{2}{*}{ Treatment } & \multirow{2}{*}{ Stage } & \multirow{2}{*}{$\begin{array}{l}\text { Sample } \\
\text { size }\end{array}$} & \multirow{2}{*}{$\begin{array}{c}\text { Age average mean } \\
\text { (sd) }\end{array}$} & \multicolumn{6}{|c|}{ Survival Rate (\%) } \\
\hline & & & & & & & 1 & 2 & 3 & 4 & 5 & 10 \\
\hline S1 & Abbasi Asl M 2015 & Colorectal Cancer & $-^{*}$ & $--^{* *}$ & 88 & $56.6(13.91)$ & 79.2 & 63.7 & 52.5 & 46.2 & 39.6 & - \\
\hline S2 & Mehrabani D 2012 & Colorectal Cancer & - & - & 243 & $\begin{array}{c}\text { Male: } 52.37(15.04) \\
\text { Female: } 58.65 \\
(13.58)\end{array}$ & 93.9 & - & 50.3 & - & 27.2 & - \\
\hline S3 & Rasouli MA 2017 & Colorectal Cancer & - & - & 335 & $\begin{array}{c}\text { Male: } 61.7(1.05) \\
\text { Female: } 60.5(1.12)\end{array}$ & 87 & 69 & 57 & 42 & 33 & - \\
\hline S4 & Zare-Bandamiri M 2016 & Colorectal Cancer & - & - & 570 & $55.8(23.6)$ & - & - & - & - & 58.5 & - \\
\hline S5 & Vakili M 2016 & Colorectal Cancer & - & - & 437 & $59.12(19 / 99)$ & 85.7 & - & - & - & 71.9 & 52.5 \\
\hline S6 & Madadizadeh F 2017 & Colorectal Cancer & - & - & 561 & $55.74(13.67)$ & - & - & - & - & 59.6 & - \\
\hline S7 & Semnani S 2016 & Colorectal Cancer & - & - & 227 & $54(5.5)$ & 71 & - & 52 & - & 44 & - \\
\hline S8 & $\begin{array}{l}\text { Asghari-Jafarabadi M } \\
2009\end{array}$ & $\begin{array}{l}\text { Rectal cancer } \\
\text { Colon cancer }\end{array}$ & - & - & $\begin{array}{l}392 \\
802\end{array}$ & $\begin{array}{l}55.03(37.63) \\
53.56(14.21)\end{array}$ & $\begin{array}{l}96.0 \\
91.7\end{array}$ & $\begin{array}{l}91.2 \\
83.7\end{array}$ & $\begin{array}{l}84.0 \\
75.9\end{array}$ & $\begin{array}{l}78.2 \\
69.0\end{array}$ & $\begin{array}{l}.76 \\
63.3\end{array}$ & - \\
\hline S9 & Zare-Bandamiri M 2017 & Colorectal Cancer & - & - & 561 & $55.7(13.7)$ & - & - & - & - & 56.8 & - \\
\hline $\mathrm{S} 10$ & Moamer S 2017 & $\begin{array}{l}\text { Colorectal Cancer } \\
\text { Colorectal Cancer } \\
\text { Colorectal Cancer } \\
\text { Colorectal Cancer }\end{array}$ & $\begin{array}{l}\text { surgery } \\
\text { surgery } \\
\text { surgery } \\
\text { surgery }\end{array}$ & $\begin{array}{l}\text { Stage } 1 \\
\text { Stage } 2 \\
\text { Stage } 3 \\
\text { Stage } 4\end{array}$ & $\begin{array}{c}372 \\
117 \\
110 \\
116 \\
29\end{array}$ & $52.69(14.39)$ & $\begin{array}{l}- \\
- \\
- \\
-\end{array}$ & $\begin{array}{l}- \\
- \\
- \\
-\end{array}$ & $\begin{array}{l}- \\
- \\
- \\
-\end{array}$ & $\begin{array}{l}- \\
- \\
- \\
-\end{array}$ & $\begin{array}{l}81 \\
85 \\
69 \\
37\end{array}$ & $\begin{array}{l}- \\
- \\
- \\
-\end{array}$ \\
\hline S11 & Akhoond, MA 2011 & $\begin{array}{l}\text { Colon cancer } \\
\text { Rectal cancer }\end{array}$ & $\begin{array}{l}- \\
-\end{array}$ & $\begin{array}{l}- \\
-\end{array}$ & $\begin{array}{l}1194 \\
802 \\
392\end{array}$ & $53.59(14 / 34)$ & $\begin{array}{l}88.7 \\
81.9\end{array}$ & $\begin{array}{l}77.9 \\
74.2\end{array}$ & $\begin{array}{l}68.5 \\
60.7\end{array}$ & $\begin{array}{l}61.4 \\
47.1\end{array}$ & $\begin{array}{l}56.8 \\
41.9\end{array}$ & - \\
\hline $\mathrm{S} 12$ & Moradi A 2009 & $\begin{array}{l}\text { Colorectal Cancer } \\
\text { ascending colon cancer } \\
\text { descending colon } \\
\text { cancer } \\
\text { sigmoid cancer } \\
\text { Rectal cancer }\end{array}$ & $\begin{array}{l}- \\
- \\
- \\
- \\
-\end{array}$ & $\begin{array}{l}- \\
- \\
- \\
- \\
-\end{array}$ & $\begin{array}{c}9,892 \\
561 \\
301 \\
1160 \\
3651\end{array}$ & $\begin{array}{l}- \\
- \\
- \\
- \\
-\end{array}$ & $\begin{array}{c}.84 \\
- \\
- \\
- \\
-\end{array}$ & $\begin{array}{c}.68 \\
- \\
- \\
- \\
-\end{array}$ & $\begin{array}{c}.54 \\
- \\
- \\
- \\
-\end{array}$ & $\begin{array}{c}.43 \\
- \\
- \\
- \\
-\end{array}$ & $\begin{array}{l}.41 \\
.61 \\
.57 \\
.50 \\
.37\end{array}$ & $\begin{array}{l}- \\
- \\
- \\
-\end{array}$ \\
\hline $\mathrm{S} 13$ & $\begin{array}{l}\text { Moghimi-Dehkordi B } \\
2008\end{array}$ & Colorectal Cancer & - & - & 1127 & $53.59(14.34)$ & 91.1 & - & 73.1 & - & .61 & 47.9 \\
\hline S14 & Saki Malehi A 2012 & Colorectal Cancer & - & - & 739 & $59 / 7(12 / 85)$ & - & - & - & - & 63.3 & - \\
\hline S15 & Ansari R, 2007 & $\begin{array}{l}\text { Colorectal Cancer } \\
\text { Transverse colon } \\
\text { cancer } \\
\text { Rectal cancer } \\
\text { Ascending colon cancer } \\
\text { Descending colon } \\
\text { cancer } \\
\text { Sigmoid cancer }\end{array}$ & $\begin{array}{l}\text { surgery } \\
\text { surgery } \\
\text { surgery } \\
\text { surgery } \\
\text { surgery } \\
\text { surgery }\end{array}$ & $\begin{array}{l}- \\
- \\
- \\
- \\
-\end{array}$ & $\begin{array}{c}226 \\
16 \\
93 \\
39 \\
55 \\
23\end{array}$ & $58.15(14.80)$ & $\begin{array}{l}.80 \\
.69 \\
.88 \\
.87 \\
.83 \\
.75\end{array}$ & $\begin{array}{l}- \\
- \\
- \\
- \\
-\end{array}$ & $\begin{array}{l}- \\
- \\
- \\
- \\
-\end{array}$ & $\begin{array}{l}- \\
- \\
- \\
- \\
-\end{array}$ & $\begin{array}{l}.49 \\
0.49 \\
0.46 \\
0.61 \\
0.62 \\
0.34\end{array}$ & $\begin{array}{l}- \\
- \\
- \\
- \\
-\end{array}$ \\
\hline S16 & Gohari MR 2011 & $\begin{array}{l}\text { Colon cancer } \\
\text { Rectal cancer }\end{array}$ & - & - & $\begin{array}{l}786 \\
204\end{array}$ & $\begin{array}{l}53.7(14.6) \\
52.4(13.7)\end{array}$ & $\begin{array}{l}.92 \\
.86\end{array}$ & - & $\begin{array}{l}.71 \\
.71\end{array}$ & - & $\begin{array}{l}.48 \\
.42\end{array}$ & - \\
\hline S17 & Omidvari S 2013 & Rectal cancer & - & - & 153 & 57.15 & - & - & - & - & 69.4 & - \\
\hline S18 & Heidarnia MA 2013 & Colon cancer & - & - & 559 & $63(11.8)$ & - & - & - & - & 68.3 & - \\
\hline S19 & Akhavan A 2014 & $\begin{array}{l}\text { Rectal cancer } \\
\text { Rectal cancer }\end{array}$ & $\begin{array}{l}- \\
\text { Surgery \& Chemo- } \\
\text { Radiotherapy }\end{array}$ & - & $\begin{array}{c}119 \\
25\end{array}$ & $\begin{array}{c}60.8 \\
-\end{array}$ & $\begin{array}{l}97 \\
-\end{array}$ & - & $\begin{array}{c}88 \\
-\end{array}$ & - & $\begin{array}{l}55 \\
85\end{array}$ & - \\
\hline S20 & Mehrkhani F 2009 & Colorectal Cancer & Surgery & - & 1090 & 50.5 & 72 & 54 & - & - & 47 & - \\
\hline S21 & Emami S 2005 & Colorectal Cancer & $\begin{array}{l}- \\
- \\
- \\
- \\
- \\
-\end{array}$ & $\begin{array}{l}- \\
- \\
- \\
- \\
-\end{array}$ & $\begin{array}{c}952 \\
586 \\
96 \\
135 \\
28 \\
107\end{array}$ & $\begin{array}{l}- \\
- \\
- \\
- \\
- \\
-\end{array}$ & $\begin{array}{l}97 \\
96 \\
96 \\
96 \\
91 \\
95\end{array}$ & $\begin{array}{l}- \\
- \\
- \\
- \\
- \\
-\end{array}$ & $\begin{array}{c}43 \\
- \\
- \\
- \\
- \\
-\end{array}$ & $\begin{array}{l}- \\
- \\
- \\
- \\
-\end{array}$ & $\begin{array}{l}21 \\
40 \\
29 \\
42 \\
29 \\
13\end{array}$ & $\begin{array}{l}- \\
- \\
- \\
- \\
- \\
-\end{array}$ \\
\hline S22 & Ahmadloo N 2005 & Colon cancer & $\begin{array}{l}\text { Surgery \& chemo- } \\
\text { therapy } \\
\text { Surgery\&Chemo- } \\
\text { Radiotherapy }\end{array}$ & $\begin{array}{l}- \\
-\end{array}$ & $\begin{array}{l}31 \\
34\end{array}$ & $\begin{array}{l}50(13) \\
46(12)\end{array}$ & $\begin{array}{l}- \\
-\end{array}$ & $\begin{array}{l}- \\
-\end{array}$ & $\begin{array}{l}86.6 \\
65.8\end{array}$ & $\begin{array}{l}- \\
-\end{array}$ & $\begin{array}{l}- \\
-\end{array}$ & $\begin{array}{l}- \\
-\end{array}$ \\
\hline S23 & Fatemi SR 2015 & $\begin{array}{l}\text { Colorectal Cancer } \\
\text { Rectal cancer } \\
\text { Colon cancer }\end{array}$ & $\begin{array}{l}\text { Surgery } \\
\text { Surgery } \\
\text { Surgery }\end{array}$ & $\begin{array}{l}- \\
- \\
-\end{array}$ & $\begin{array}{c}107 \\
- \\
-\end{array}$ & $53.50(12.68)$ & $\begin{array}{l}- \\
- \\
-\end{array}$ & $\begin{array}{l}- \\
- \\
-\end{array}$ & $\begin{array}{l}- \\
- \\
-\end{array}$ & $\begin{array}{l}- \\
- \\
-\end{array}$ & $\begin{array}{l}73.8 \\
70.0 \\
75.9\end{array}$ & $\begin{array}{l}- \\
- \\
-\end{array}$ \\
\hline 37 & Aryaie M 2013 & $\begin{array}{l}\text { Colorectal Cancer } \\
\text { Colorectal Cancer }\end{array}$ & & $\begin{array}{c}\text { Stage } 1 \\
\text { Stage } 2 \& 3\end{array}$ & $\begin{array}{c}42 \\
105\end{array}$ & $53.6(1.1)$ & $\begin{array}{c}1 \\
92.4\end{array}$ & - & - & $\begin{array}{l}- \\
-\end{array}$ & $\begin{array}{l}80.6 \\
51.5\end{array}$ & $\begin{array}{l}- \\
-\end{array}$ \\
\hline S24 & Payandeh M 2016 & Colorectal Cancer & - & - & 186 & $54.27) 13.24($ & - & - & - & - & 84.6 & - \\
\hline S25 & Vakili M 2014 & Colorectal Cancer & - & - & 604 & $58.9) 19.99$ & 72.3 & - & - & - & 50.1 & 39.8 \\
\hline S26 & Allemani C 2018 & $\begin{array}{l}\text { Colon cancer } \\
\text { Rectal cancer }\end{array}$ & - & - & $\begin{array}{c}216 \\
58\end{array}$ & - & $\begin{array}{l}- \\
-\end{array}$ & - & - & - & $\begin{array}{l}29.1 \\
26.2\end{array}$ & $\begin{array}{l}- \\
- \\
\end{array}$ \\
\hline
\end{tabular}

*Unidentified treatment; ${ }^{* *}$ Unrecognized stage 
Table 2. Results of meta-analysis for SR of CC in subgroups according to topography codes and treatment: number of observations, heterogeneity test, tests for publication, pooled SR $(95 \% \mathrm{CI})$ and meta-regression results.

\begin{tabular}{|c|c|c|c|c|c|}
\hline Outcomes & $\mathbf{N}$ & ${ }^{*} \mathbf{I}^{2}$ & ${ }^{\# \# \text { Publication bias }}$ & Pooled 5-YSR (95\%CI) & $\begin{array}{c}\text { Meta-regression coefficient } \\
\text { (p-value) }\end{array}$ \\
\hline \multicolumn{6}{|l|}{ Colorectal cancer } \\
\hline Total & 23 & 98.6 & 0.895 & $0.559(0.492,0.626)$ & \\
\hline Surgery & 7 & 96.8 & 0.881 & $0.636(0.496,0.776)$ & $0.11(0.196)$ \\
\hline Unrecognized & 16 & 98.8 & 1.000 & $0.526(0.446,0.606)$ & Reference \\
\hline \multicolumn{6}{|l|}{ Colon cancer } \\
\hline Total & 5 & 95.6 & 1.000 & $0.531(0.410,0.653)$ & \\
\hline Surgery & 0 & - & - & - & $\Omega$ \\
\hline Unrecognized & 5 & 95.6 & 1.000 & $0.531(0.410,0.653)$ & \\
\hline \multicolumn{6}{|l|}{ Rectal cancer } \\
\hline Total & 10 & 97.9 & 0.095 & $0.516(0.411,0.621)$ & \\
\hline Surgery & 2 & 94.9 & 0.317 & $0.652(0.270,1.034)$ & $0.17(0.310)$ \\
\hline Unrecognized & 8 & 98.1 & 0.099 & $0.486(0.372,0.600)$ & Reference \\
\hline \multicolumn{6}{|c|}{ Ascending colon cancer } \\
\hline Total & 3 & 98.7 & 0.602 & $0.448(0.089,0.806)$ & \\
\hline Surgery & 1 & - & - & $0.610(0.457,0.763)$ & $0.24(0.672)$ \\
\hline Unrecognized & 2 & 99.2 & 0.317 & $0.371(0.100,0.841)$ & Reference \\
\hline \multicolumn{6}{|c|}{ Descending colon cancer } \\
\hline Total & 3 & 81.7 & 0.602 & $0.532(0.419,0.645)$ & \\
\hline Surgery & 1 & - & - & $0.620(0.492,0.748)$ & $0.12(0.546)$ \\
\hline Unrecognized & 2 & 88.4 & 0.317 & $0.498(0.351,0.645)$ & Reference \\
\hline \multicolumn{6}{|c|}{ Transverse colon cancer } \\
\hline Total & 2 & 42.6 & 0.317 & $0.354(0.215,0.493)$ & \\
\hline Surgery & 1 & - & - & $0.490(0.245,0.735)$ & $\Omega$ \\
\hline Unrecognized & 1 & - & - & $0.290(0.122,0.458)$ & \\
\hline \multicolumn{6}{|l|}{ Sigmoid cancer } \\
\hline Total & 3 & 90.3 & 0.602 & $0.385(0.219,0.551)$ & \\
\hline Surgery & 1 & - & - & $0.340(0.146,0.534)$ & $-0.06(0.819)$ \\
\hline Unrecognized & 2 & 94.6 & 0.317 & $0.400(0.194,0.605)$ & Reference \\
\hline \multicolumn{6}{|l|}{ Total } \\
\hline Total & 49 & 98.3 & 0.398 & $0.524(0.481,0.566)$ & \\
\hline Surgery & 13 & 94.4 & 0.329 & $0.607(0.507,0.707)$ & $0.111(0.057)$ \\
\hline Unrecognized & 36 & 98.0 & 0.327 & $0.496(0.448,0.544)$ & Reference \\
\hline
\end{tabular}

N: Number of Observations; ${ }^{*}$ Heterogeneity Index: Value upper $50 \%$ needs Random effect method of estimation; Meta-regression coefficient showed difference of Five-Year-SR in surgery vs. unrecognized studies; $\Omega$ : Insufficient Data; ${ }^{* *}$ Egger test of publication bias which test small-study effects.

The lowest 5-YSR was observed in the study of Emami et al. for ascending colon cancer - $13 \%(95 \%$ CI: 7,19$)$ and the highest SR was observed in the study of Moamer et al. for CC - 85\% (95\% CI: 78, 92) [S10, S20]. Random-effect pooled 5-YSR was estimated - 52\% (95\% CI: 48, 57), regardless the type of treatment and stage of the disease.

Heterogeneity and publication bias. According to I-square test significant heterogeneity (except for Transverse colon cancer I-squared lower 50\%), were obtained. In order to overcome this issue, subgroup analysis based on the topography codes of CC, type of treatment and type of data source was conducted. Thanks to subgroup analysis for topography codes, the 5-YSR of patients with colorectal, colon, rectal, ascending colon, descending colon, transverse colon and sigmoid cancers were 56\%, 53\%, 52\% 45\%, 53\%, 37\% and $38 \%$, respectively (Supplementary Figure S1). The 5-YSR of post-operative and unrecognized treatment of CC patients were $64 \%$ (95\% CI: 50, 78) and 53\% (95\% CI: 45, 61), respectively (Table 2, Supplementary Figure S1). Meta-regression analysis was fitted to estimate the difference between rates of survival in surgery versus unrecognized treatment. Results showed higher rate of survival in colorectal $(11 \%, \mathrm{p}=0.196)$, rectal $(17 \%, \mathrm{p}=0.310)$, ascending colon $(0.24 \%, \mathrm{p}=0.672)$, descending colon $(0.12 \%, \mathrm{p}=0.546)$ (Table 2, Figure 2). In addition, subgroup analysis was conducted by type of data sources. Pooled 5-YSR were estimated - 75\% (95\% CI: 54, 95), 
55\% (95\% CI: 50, 60) and 45\% (95\% CI: 37, 53) in Oncology Center, Cancer Registry center and Hospital Records, respectively. Results of meta-regression revealed higher rates of survival in Oncology Center $(29 \%, \mathrm{p}=0.005)$ and cancer registry center $(9.6 \%, \mathrm{p}=0.059)$ compared to hospital records (Table 3 and Figure 2). No publication bias was found between the studies in various subgroups based on Begg's test.

Sensitivity analysis. Sensitivity analysis was run to explore the source of heterogeneity. In this process, we identified two heterogeneous studies [S12, S21]. After exclusion of them, results for random effect pooled 5-YSR was estimated: 53\% (95\% CI: 49, 58). CC was also estimated: 58\% (95\%CI: 52, 64), they did not change significantly (Figure 3 ).
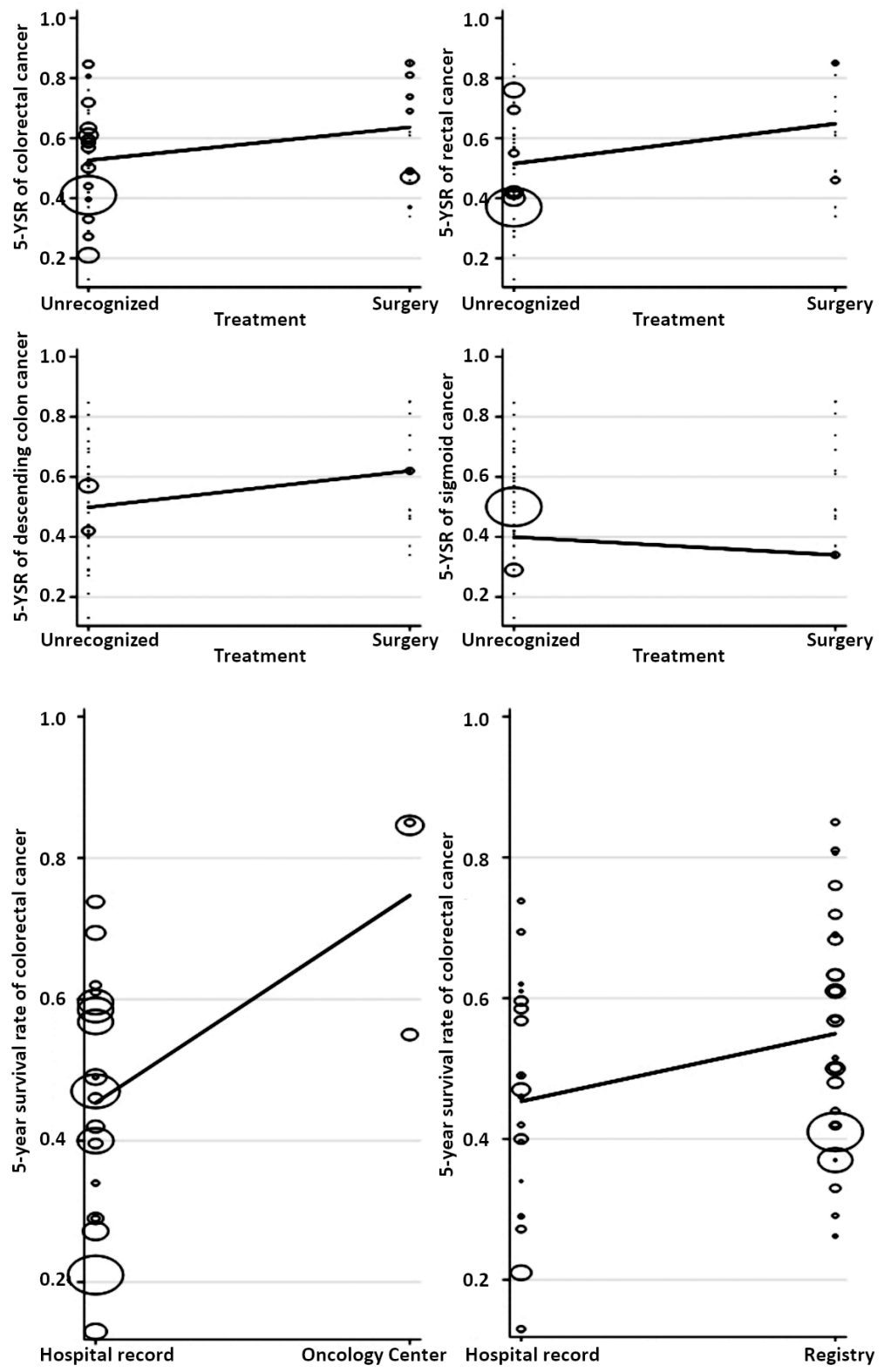

Figure 2. Bubble plots show the differences between 5-YSR of colorectal cancer in subgroups of type of treatment and source of data. 
Table 3. Results of meta-analysis for SR of CC in subgroups according to type of data source: number of observations, heterogeneity test, tests for publication bias, pooled SR $(95 \% \mathrm{CI})$ and meta-regression results.

\begin{tabular}{lccccc}
\hline Subgroups & $\mathbf{N}$ & ${ }^{*} \mathbf{I}^{2}$ & ${ }^{\# *}$ Publication bias & Pooled 5-YSR (95\%CI) & $\begin{array}{c}\text { Meta-regression coefficient } \\
(\mathbf{p}-\mathrm{value})\end{array}$ \\
\hline Oncology Center & 3 & 97 & 0.312 & $0.747(0.542,0.953)$ & $0.293\left(0.005^{*}\right)$ \\
Cancer Registry center & 26 & 97 & 0.653 & $0.550(0.498,0.603)$ & $0.096(0.059)$ \\
Hospital Records & 20 & 98 & 0.697 & $0.454(0.375,0.532)$ & Reference \\
\hline
\end{tabular}

N: Number of observations; "Heterogeneity Index: Value upper 50\% needs Random effect method of estimation; Meta-regression coefficient shows difference of Five-Year-SR in Oncology center and cancer registry vs hospital records studies; ${ }^{\# *}$ Egger test of publication bias which test small-study effects; ${ }^{*}$ Statistical significance level $\mathrm{p}<0.05$.

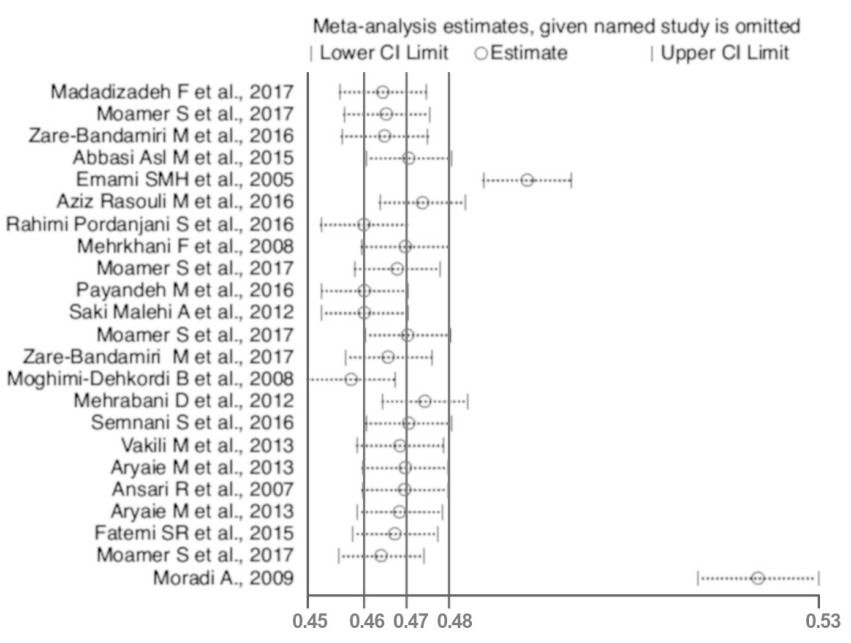

Figure 3. Forest of 5-year survival rate of colorectal cancer, plot obtained from Sensitivity analysis.

Our meta-analysis study showed that the 5-YSR of colon cancer patients $(53 \%$; $95 \%$ CI: 41,65$)$ was higher than that of rectal cancer patients (52\%; 95\% CI: 41, 62). Higher SR of colon cancer patients, compared to rectal cancer patients, has been confirmed in the other studies [11-13]. According to subgroup analysis, the pooled estimation of 5-YSR of postoperative CC patients was $64 \%$ (95\% CI: 50,78 ), which is $11 \%$ higher than the SR of those with an unspecific treatment status - 53\% (95\% CI: 45, 61), although meta-regression could not find statistically significant difference $(\mathrm{p}=0.196)$.

In this study, the pooled estimation of 5-YSR of patients with CC was determined to be $52 \%$ (95\% CI: 48,57$)$. While meta-regression analysis found no statistically significant differences between studies with surgery and unrecognized treatment, in most of them an increased rate was in the surgery group. In addition, subgroup analysis for type of data source revealed a higher rate of survival in oncology and cancer registry center than hospital-based ones. This incline may happen thanks to the health status of patients, which meant that poorer conditions referred to hospital.

To reduce heterogeneity, subgroup analyses were conducted based on the topography of cancer, treatment status and type of data. No changes in the values of heterogeneity were observed while meta-regression results showed a significant difference in categories of type of data (oncology center compared to hospital records) [14]. Sensitivity analysis provided no significant results as well. Factors such as age, gender, education, socioeconomic status and stage of disease can affect SRs of CC. Therefore, the heterogeneity and differences reported in these studies could be caused by the differences between studied patients $[S 4,15]$. A minority of researches have included treatment status and stage of disease in their study to obtain SRs. The available evidence showed the more locally advanced stage of CC occurs below 50 years in Iran which ended up with poor prognosis. The CC growth among Iranian population, as well as younger age distributions and lower SRs necessitate immediate prevention actions [16]. Considering the association between colon polyps and the incidence of CC, prevention strategies and the effectiveness of health systems, such as detection of precancerous polyps through screening, are recommended in order to prevent this disease [17]. In addition, early detection, finding patients at initial stages of the disease, and early treatment can effectively increase the SR of such patients $[18,19]$.

The strengths of this systematic review and meta-analysis include the use of national studies to estimate pooled SR of CC in Iran for the first time, which could provide essential information or the use of policymakers in the realm of health. The main limitation of this study was an insufficient number of publications in considering subgroups, therefore, heterogeneity of the results could not be overcome appropriately. In addition, many studies did not clearly specify the type of treatment, which patients were undertaken.

Supplementary information is available in the online version of the paper.

Acknowledgements: This research received no specific grant from any funding agency in the public, commercial, or not-forprofit sectors.

\section{References}

[1] MA X, YU H. Global burden of cancer. Yale J Biol Med 2006; 79: 85-94. 
[2] FITZMAURICE C, ALLEN C, BARBER RM, BARREGARD L, BHUTTA ZA et al. Global, regional, and national cancer incidence, mortality, years of life lost, years lived with disability, and disability-adjusted life-years for 32 cancer groups, 1990 to 2015: a systematic analysis for the global burden of disease study. JAMA Oncol 2017; 3: 524-548 https:// doi.org/10.1001/jamaoncol.2016.5688

[3] Center for Disease Control and Prevention, Noncommunicable Deputy Cancer Office. [Iranian Annual National Cancer Registration Report 2014 - 2015]. Ministry of Health and Medical Education; Tehran, Iran, 2018. ISBN 978-600-333346-8

[4] FARHOOD B, GERAILY G, ALIZADEH A. Incidence and Mortality of Various Cancers in Iran and Compare to Other Countries: A Review Article. Iran J Public Health 2018; 47: 309-316.

[5] ENAYATRAD M, MIRZAEI M, SALEHINIYA H, KARIMIRAD MR, VAZIRI S et al. Trends in incidence of common cancers in Iran. Asian Pac J Cancer Prev 2016; 17: 3942. https://doi.org/10.7314/apjcp.2016.17.s3.39

[6] MARIOTTO AB, NOONE AM, HOWLADER N, CHO H, KEEL GE et al. Cancer survival: an overview of measures, uses, and interpretation. J Natl Cancer Inst Monogr 2014; 2014: 145-186. https://doi.org/10.1093/jncimonographs/ lgu024

[7] LibERATI A, ALTMAN DG, TETZLAFF J, MULROW C, GOTZSCHE PC et al. The PRISMA statement for reporting systematic reviews and meta-analyses of studies that evaluate health care interventions: explanation and elaboration. PLoS Med 2009; 6: e1000100. https://doi.org/10.1371/journal. pmed. 1000100

[8] DUVAL S, TWEEDIE R. Trim and fill: a simple funnel-plotbased method of testing and adjusting for publication bias in meta-analysis. Biometrics 2000; 56: 455-463. https://doi. org/10.1111/j.0006-341X.2000.00455.X

[9] ALLEMANI C, RACHET B, WEIR HK, RICHARDSON LC, LEPAGE $C$ et al. Colorectal cancer survival in the USA and Europe: a CONCORD high-resolution study. BMJ Open 2013; 3: e003055. https://doi.org/10.1136/bmjopen-2013-003055
[10] VERDECCHIA A, FRANCISCI S, BRENNER H, GATTA G, MICHELI A et al. Recent cancer survival in Europe: a 2000-02 period analysis of EUROCARE-4 data. Lancet Oncol 2007; 8: 784-796. https://doi.org/10.1016/S14702045(07)70246-2

[11] LEE YC, LEE YL, CHUANG JP, LEE JC. Differences in survival between colon and rectal cancer from SEER data. PLoS One 2013; 8: e78709. doi:10.1371/journal.pone.0078709

[12] WILKES G, HARTSHORN K. Clinical update: colon, rectal, and anal cancers. Semin Oncol Nurs 2012; 28: e1-e22. https://doi.org/10.1016/j.soncn.2012.09.012

[13] BERRINO F, DE ANGELIS R, SANT M, ROSSO S, BIELSKA-LASOTA M et al. Survival for eight major cancers and all cancers combined for European adults diagnosed in 199599: results of the EUROCARE-4 study. Lancet Oncol 2007; 8: 773-783. https://doi.org/10.1016/S1470-2045(07)70245-0

[14] GRINDEM H, MANSOURNIA MA, ØIESTAD BE, ARDERN CL. Was it a good idea to combine the studies? Why clinicians should care about heterogeneity when making decisions based on systematic reviews. Br J Sports Med 2019; 53: 399-401. https://doi.org/10.1136/bjsports-2018-099516

[15] HENRY KA, NIU X, BOSCOE FP. Geographic disparities in CC survival. Int J Health Geogr 2009; 8: 48. https://doi. org/10.1186/1476-072X-8-48

[16] MAHMODLOU R, MOHAMMADI P, SEPEHRVAND N. Colorectal cancer in northwestern Iran. ISRN Gastroenterol 2012; 2012: 968560. https://doi.org/10.5402/2012/968560

[17] NEWCOMB PA, NORFLEET RG, STORER BE, SURAWICZ TS, MARCUS PM. Screening sigmoidoscopy and colorectal cancer mortality. JNCI: J Natl Cancer Inst 1992; 84: 1572-1575. https://doi.org/10.1093/jnci/84.20.1572

[18] BRENNER H, JANSEN L, ULRICH A, CHANG-CLAUDE J, HOFFMEISTER M. Survival of patients with symptomand screening-detected colorectal cancer. Oncotarget 2016; 7: 44695-44704. https://doi.org/10.18632/oncotarget.9412

[19] MENGUAL-BALLESTER M, PELLICER-FRANCO E, VALERO-NAVARRO G, SORIA-ALEDO V, GARCIAMARIN JA et al. Increased survival and decreased recurrence in colorectal cancer patients diagnosed in a screening programme. Cancer Epidemiol 2016; 43: 70-75. https://doi. org/10.1016/j.canep.2016.06.003 